\title{
Diagnostic Dilemma In Diagnosing Rare Cause Of Protein Losing Enteropathy:Waldmann's Disease
}

\author{
Sarita Prajapati ${ }^{1}$, Sujan Bohara ${ }^{2}$, Gaurab Mainali ${ }^{3}$, Samikshya Karki ${ }^{3}$, Sharan Thapa ${ }^{4}$, \\ and Nirjala Aryal ${ }^{1}$ \\ ${ }^{1}$ Birendra Military Hospital \\ ${ }^{2}$ Nepal Mediciti \\ ${ }^{3}$ Nepalese Army Institute of Health Sciences College of Medicine \\ ${ }^{4}$ Lekhnath City Hospital Pvt. Ltd.
}

March 1, 2022

\begin{abstract}
Waldmann's disease / Primary intestinal lymphangiectasia, is an unusual cause of protein losing enteropathy primarily characterized by lymphopenia, hypoalbuminemia and hypogammaglobulinemia. However, variable clinical presentation results in diagnostic dilemma and effective management. We present a toddler diagnosed with Waldmann's disease managed with high protein diet and medium chain triglycerides supplementation.
\end{abstract}

\section{Diagnostic Dilemma In Diagnosing Rare Cause Of Protein Losing Enteropathy:Waldmann's Disease}

Sarita Prajapati ${ }^{1}$, Sujan Bohara ${ }^{2}$, Gaurab Mainali ${ }^{3}$, Samikshya Karki $^{3}$, Sharan Thapa ${ }^{4}$, Nirjala Aryal ${ }^{1}$

${ }^{1}$ Department of Pediatrics, Birendra Military Hospital, Chhauni, Kathmandu, Nepal.

2 Department of General and Gastrointestinal Surgery, Nepal Mediciti Hospital, Lalitpur, Nepal

${ }^{3}$ Nepalese Army Institute of Health Sciences, Kathmandu, Nepal

${ }^{4}$ Lekhnath City Hospital Private Limited, Pokhara, Nepal

Corresponding Author: Dr. Sujan Bohara, Nepal Mediciti Hospital, Lalitpur, Nepal

Email address : mjsujan777@gmail.com Phone : +977-9860103009

Disclosures can be found in Additional Information at the end of the article

Abstract Waldmann's disease / Primary intestinal lymphangiectasia, is an unusual cause of protein losing enteropathy pri

Keywords: Waldmann's disease, protein losing enteropathy, intestinal lymphangiectasia, medium chain triglycerides

Introduction

Intestinal lymphangiectasis is rare cause of protein losing enteropathy characterized by diffuse or local dilatation of the enteric lymphatics mostly presenting with feature of hypoproteinemia leading to bilateral lower limb edema, ascites or rarely anasarca. Waldmann and Schwabb in 1961 reported this rare disease 
with intestinal protein loss leading to hypoproteinemia and anasarca.(1) Intestinal lymphangiectasia can be either primary (idiopathic) or secondary. Primary intestinal lymphangiectasia usually occurs in children and adolescents, due to the congenital deformity of the small bowel lymphatic system, whereas secondary intestinal lymphangiectasia is more often seen in adults and occurs secondary to an elevated lymphatic pressure such as in lymphoma, systemic lupus erythematosus, inflammatory bowel disease, malignancies, constrictive pericarditis, and cardiac surgery.(2)

Primary intestinal lymphangiectasia (PIL) is a congenital disorder of the lymphatic system characterized by marked ectasia of the lymphatic vessels resulting in obstruction and leakage of lymph fluid.(3) The time of presentation may be vary from early childhood to adult with different presentation. Diagnosis of primary intestinal lymphangiectasia done with histo-pathological examination of biopsy taken from the involved part of the intestine via demonstrating dilated lymphatics in mucosa, sub mucosa and serosa in the absence of coexistent inflammation. $(2,4,5)$ Treatment vary from symptomatic management like nutritional supplement to advanced surgical treatment. $(2,3)$

We herein reported a 3 years old toddler with primary intestinal lymphangiectasia presented with periorbital edema, abdominal distension with bilateral pitting edema i.e generalized anasarca managed with medium chain triglycerides, high protein diet and multivitamin with fat soluble vitamin supplementation.

\section{Case Presentation}

We presented a three-years-old female toddler, product of full term normal vaginal delivery born out of nonconsanguineous marriage, presented in our pediatric department with complaint of abdominal distension since 1 month and periorbital as well as bilateral limb swelling since 3 days. There was no history of passing of red color urine, urgency, frequency, painful micturition, cough, altered sensorium, abnormal body movement, headache, photophobia, rash, joint pain. Her feeding and activities were normal. There was no history of recurrent infections, chronic or significant genetic diseases in her family.

Her anthropometric and nutritional assessment affirmed as follows; Height- $91 \mathrm{~cm}$, Weight-14.5 kg, HC- 47 $\mathrm{cm}$, MUAC-13 cm, Weight for age- 0 to $-1 \mathrm{SD}$, Height for age- 0 to $+1 \mathrm{SD}$, Weight for height- +1 to $+2 \mathrm{SD}$

Physical examination revealed bilateral lower limb pitting edema with periorbital edema and distended abdomen (with abdominal girth of $56 \mathrm{~cm}$ at umbilicus level) with flank fullness, slit-like centrally placed umbilicus along with presence of fluid thrill and shifting dullness but hepatosplenomegaly was not noted. Other systemic finding and vital parameters were within normal limit.

Patient had been admitted with provisional diagnosis of nephrotic syndrome and further work-up was done.Initial laboratory reports revealed anemia ( $\mathrm{Hb} 11.2 \mathrm{gm} / \mathrm{dl}$ ), lymhopenia (15\%), thrombocytosis $\left(668000 / \mathrm{mm}^{3}\right)$, hypocalcemia $(8 \mathrm{mg} / \mathrm{dl})$, normal C-reactive protein, liver function test, renal function test and electrolytes, lipid profile and thyroid function test. Peripheral blood smears showed microcytic hypochromic anemia with thrombocytosis. Iron profile and fat soluble vitamin assessment showed decreased in total iron level $(54 \mathrm{mcg} / \mathrm{dl})$ and decreased vitamin D (13.6 ng/dl) respectively. Ultrasonography of whole abdomen showed moderate ascities, multiple enlarged mesenteric lymph nodes with mildly diffused mucosal thickening of bowel loop but normal shape, outline and echo-texture of hepatic, renal and splenic structure.Urinalysis, culture and sensitivity and a 24 - hour urinary protein $(2.7 \mathrm{gm} / \mathrm{dl})$ were normal which ruled out the renal cause. Transthoracic echocardiography appeared normal thus ruling out cardiac cause through clinical examination and echocardiography. Ascitic fluid analysis revealed triglycerides rich chylous ascites (exudative type). Upper gastrointestinal endoscopy (Fig. 1) showed normal mucosa over the esophagogastric region along with white patches or streaks over the $1^{\text {st }}$ and $2^{\text {nd }}$ part of duodenum. $\mathrm{H}$ and $\mathrm{E}$ staining of Duodenal biopsy (Fig. 2) showed tissue lined by columnar epithelium forming crypts and villi with apical goblet cells and multiple dilated lymphatic channels in lamina propria with inflammatory infiltrates comprising of lymphocytes, plasma cells and eosinophils suggestive of intestinal lymphangiectasia. Colonoscopy showed no any distint mucosal abnormalities. Other biological workup like parasitological stool examination, Serological test (HIV, CMV), and tuberculosis assessment appears normal. ANA, anti-double stranded DNA and IgA, anti-tissue transglutaminase were negative. Serum electrophoresis revealed decrease in IgG serum 
protein with normal other types of proteins.

Considering all the clinical history examination and investigations, a diagnosis of primary intestinal lymphangiectasia (Waldmann's Disease) was considered. Thereafter, child was treated with human albumin infusion as a protein supplements (@ $1 \mathrm{gm} / \mathrm{kg}, 75 \mathrm{ml}$ over 3 hours once a day for 3 days) with furosemide, multivitamins including fat-soluble vitamin, oral iron (syrup ferrofolic @ $4 \mathrm{mg} / \mathrm{kg} /$ day), calcium supplementation (syrup calvit @ $40 \mathrm{mg} / \mathrm{kg} /$ day) and MCT (Medium Chain Triglyceride) based oils.She responded to the treatment dramatically along with gradual stabilization and improvement in her clinical conditions and discharged on the $3^{\text {rd }}$ week after her admission along with advice on intake of protein rich diet, low fat diet and MCT oil based food supplementation on her diet as a lifelong dietary therapy.

Regular follow up was done once a month for first 3 months and then every three monthly from the diagnosis made till 18 months of diagnosis. Follow up visit showed satisfactory result, as child physical growth was not hampered, no any symptoms developed during follow up period and investigation were within normal limit.

\section{Discussion}

Waldmann et al. first described 18 cases of "idiopathic hypercatabolic hypoproteinemia in 1961.(1) Intestinal lymphangiectasia is an uncommon but important cause of protein loss intolerance and is characterized by poor lymph drainage in small intestines associated with enlarged lymphatic channels. The prevalence of clinically PIL is not known. $(2,6)$ It occurs mainly in children and is equally common among both sexes. $(6,7)$ Although most cases are sporadic, Le Bougeant et al reported rare familial forms of Waldmann's disease. $(8,9)$

Primary and secondary, are the two types of intestinal lymphangiectasia described in literature according to cause of the disease. In primary lymphangiectasia, there is no predisposing condition to increased lymph pressure and is probably caused by congenital anatomical malformation of the lymphatic system. PIL is associated with Yellow nail syndrome, Klippel-trenaunay-Weber syndrome, Von Recklinghausen, Noonan, Turner and Hennekam.(1-4) There is no given associated disease in our case. Hokari et al. reported inconsistently changed expressions of regulatory molecules for lymphangiogenesis in the duodenal mucosa of PIL patients.(10) Secondary intestinal lymphangiectasia is an acquired disease characterized by an increased leakage of the intestinal lymph, secondary to an increase in pressure due to obstruction in the lymphatics. (2)

The diagnosis is based on a set of clinical, biological, radiological, endoscopic and histopathological. The disease is characterized by hypoproteinemia, lymphocytopenia, edema and ascites. Clinical manifestations may be presented as asymptomatic or fatigue, lower abdominal pain, edema, chylothorax, chronic diarrhea, ascites.(1-6,8) Iron deficiency anemia and hypocalcemic tetany can be observed.(2,8) Supporting the fact, our case also had micronutrient deficiency of Iron and calcium /Vitamin D3. Along with above clinical features, Stool analysis may show steatorrhea and increased $\alpha$ anti trypsin clearance $(>24 \mathrm{ml} /$ day $) \cdot(2,8,11)$ PIL diagnosis is confirmed by the presence of intestinal lymphangiectasia based on endoscopic findings with the corresponding histology of intestinal biopsy specimens.(12) . Histological examination of duodenum-jejunum and ileum biopsies confirms the presence of lacteal juice, dilated mucosal (from moderate to severe) and submucosal lymphatic vessels (and also in the serosa) with polyclonal normal plasma cells.(2)(11) Martins et. al reported an enteroscopy showing white spots with a "snowflake" appearance, which are the typical findings of intestinal lymphangiectasia.(8) Sometimes, endoscopy may be negative when there is segmental or localized lesion. In that cases, videocapsule endoscopy is a useful tool to detect the presence of intestinal lymphangiectasia and to specify its localization. (2) Exclusion of secondary causes of intestinal lymphangiectasia like erosive and non-erosive intestinal disorders, conditions involving mesenteric lymphatic obstruction and cardiovascular disorders that increase central venous pressure, is necessary for definitive diagnosis of Waldmann's disease. (8)

A wide range of presenting symptoms provide a challenge to appropriate therapy. The treatment protocol depends on presenting symptoms localization of disease and associated complications. The treatment is based on a diet free of long-chain lipids, enriched with protein and medium-chain triglycerides. $(2-8,11)$ But, 
Ballinger et al, stated that it doesn't always give good results.(13) .In literature reported by Aoyagi et al, In patients not responding to a low fat diet, enteral nutritional therapy (elemental, semi-elemental and polymeric diets) may be required.(14) It was reported that somatostatins such as octreotide can decrease triglyceride absorption. $(2,3,8,13)$ Antiplasmin therapy may have some role when fibrinolysis is increased.(2,11) Surgical treatment is helpful in localized intestinal involvement, intestinal obstruction. $(2,8)$

\section{Conclusions}

Rareity and variable presentation of disease can led into dilemma in diagnosing the disease and management. Clinician should be aware about this rare disease and consider as one of the differential whenever patient clinically presents with anasarca, lymphedema with peri-orbital puffiness. Though rare, it can be managed early and effectively with dietary therapy and nutritional supplementation.

\section{Abbreviations:}

1. HC- Head circumference

2. MUAC- Mid Upper Arm Circumference

3. SD- Standard Deviation

4. $\mathrm{mg} / \mathrm{dl}$ : milligram per deciliter

5. gm/dl: gram per deciliter

6. $\mathrm{mm}^{3}$ : cubic millimeter

7. mcg/dl: microgram per deciliter

8. ng/dl: nanogram per deciliter

9. H \& E: Hematoxylin and Eosin

10. ANA: Anti-Nuclear Antibody

11. IgA: Immunoglobulin A

12. IgG: Immuonglobulin G

\section{Additional Information}

\section{Disclosures}

\section{Conflicts of interest: None}

2. Ethical approval: Study did not include experiments on humans or animals

3. Consent: For publication of this case report, informed written consent was taken from patient parents

4. Source of funding: Authors did not receive any grants for this study

\section{Authors Contributions:}

SP contributed on collection of the case information; SP, SB, GM, SK and ST contributed on reviewing the literature, designing and writing the manuscript. SB, SK and GM also contributed on revising, re-editing and reshaping the manuscript. NA established the diagnosis and revised the manuscript critically for important intellectual content. All authors read and approved the final version of the manuscript.

\section{References}

1. Waldmann Ta, Steinfeld Jl, Dutcher Tf, Davidson Jd, Gordon Rsj. The role of the gastrointestinal system in "idiopathic hypoproteinemia". Gastroenterology. 1961 Sep;41:197-207. 
2. Vignes S, Bellanger J. Primary intestinal lymphangiectasia (Waldmann's disease). Orphanet J Rare Dis [Internet]. 2008 Feb 22;3:5. Available from: https://pubmed.ncbi.nlm.nih.gov/18294365

3. Faul JL, Berry GJ, Colby T V, Ruoss SJ, Walter MB, Rosen GD, et al. Thoracic lymphangiomas, lymphangiectasis, lymphangiomatosis, and lymphatic dysplasia syndrome. Am J Respir Crit Care Med. 2000 Mar;161(3 Pt 1):1037-46.

4. Roberts SH, Douglas AP. Intestinal lymphangiectasia: the variability of presentation. A study of five cases. Q J Med. 1976 Jan;45(177):39-48.

5. Hilliard RI, McKendry JB, Phillips MJ. Congenital abnormalities of the lymphatic system: a new clinical classification. Pediatrics. 1990 Dec;86(6):988-94.

6. El Haddar Z, Sbia Y, Rkain M, Benajiba N, Babakhouya A. Complicated primary intestinal lymphangiectasia (Waldmann's disease) in a child successfully treated with octreotide: A case report from a low-resource setting. Ann Med Surg [Internet]. 2021 Jul 29;68:102588. Available from: https://pubmed.ncbi.nlm.nih.gov/34401115

7. Tift WL, Lloyd JK. Intestinal lymphangiectasia. Long-term results with MCT diet. Arch Dis Child [Internet]. 1975 Apr;50(4):269-76. Available from: https://pubmed.ncbi.nlm.nih.gov/50050

8. Martins CR, Gagnaire A, Rostain F, Lepage C. Waldmann's disease: a rare cause of protein losing enteropathy in an adult patient. Rev Esp enfermedades Dig organo Of la Soc Esp Patol Dig. 2017 May;109(5):385-8.

9. Le Bougeant P, Delbrel X, Grenouillet M, Leou S, Djossou F, Beylot J, et al. [Familial Waldmann's disease]. Ann Med Interne (Paris). 2000 Oct;151(6):511-2.

10. Hokari R, Kitagawa N, Watanabe C, Komoto S, Kurihara C, Okada Y, et al. Changes in regulatory molecules for lymphangiogenesis in intestinal lymphangiectasia with enteric protein loss. J Gastroenterol Hepatol. 2008 Jul;23(7 Pt 2):e88-95.

11. Levitt DG, Levitt MD. Protein losing enteropathy: comprehensive review of the mechanistic association with clinical and subclinical disease states. Clin Exp Gastroenterol [Internet]. 2017 Jul 17;10:147-68. Available from: https://pubmed.ncbi.nlm.nih.gov/28761367

12. Lai Y, Yu T, Qiao X-Y, Zhao L-N, Chen Q-K. Primary intestinal lymphangiectasia diagnosed by double-balloon enteroscopy and treated by medium-chain triglycerides: a case report. J Med Case Rep. 2013 Jan;7:19.

13. Ballinger AB, Farthing MJ. Octreotide in the treatment of intestinal lymphangiectasia. Eur J Gastroenterol Hepatol. 1998 Aug;10(8):699-702.

14. Aoyagi K, Iida M, Matsumoto T, Sakisaka S. Enteral nutrition as a primary therapy for intestinal lymphangiectasia: value of elemental diet and polymeric diet compared with total parenteral nutrition. Dig Dis Sci. 2005 Aug;50(8):1467-70.

\section{Hosted file}

Figures.docx available at https://authorea.com/users/462878/articles/558177-diagnosticdilemma-in-diagnosing-rare-cause-of-protein-losing-enteropathy-waldmann-s-disease 